\title{
Transmission of Pseudomonas aeruginosa epidemic strain from a patient with cystic fibrosis to a pet cat
}

\author{
K Mohan, ${ }^{1} \mathrm{~J}$ L Fothergill, ${ }^{2} \mathrm{~J}$ Storrar, ${ }^{3}$ M J Ledson, ${ }^{1}$ C Winstanley, ${ }^{2}$ M J Walshaw ${ }^{1}$
}

${ }^{1}$ The Adult Cystic Fibrosis Unit, The Cardiothoracic Centre NHS Trust, Liverpool, UK; ${ }^{2}$ Division of Medical Microbiology, University of Liverpool, Liverpool, UK; ${ }^{3}$ The Storrar Practice, Chester, UK

Correspondence to:

Dr K Mohan, The Adult Cystic Fibrosis unit, The Cardiothoracic Centre NHS Trust, Thomas

Drive, Liverpool L14 3PE, UK kamleshmohan@hotmail.com

Received 23 October 2007 Accepted 31 December 2007

\section{ABSTRACT}

Chronic infection with Pseudomonas aeruginosa is common in cystic fibrosis (CF) and certain strains are more transmissible and virulent than others. Of these, the Liverpool Epidemic Strain (LES) is highly transmissible and cross infection has been reported between patients with CF and healthy non-CF relatives. However, the risk of transmission from humans to animals is unknown. The first report of interspecies transmission of the LES strain of $P$ aeruginosa from an adult patient with CF to a pet cat is described. This development further complicates the issue of infection control policies required to prevent the spread of this organism.

A previously well 5-year-old neutered male Egyptian Mau cat presented with a 6 week history of intermittent serous nasal discharge and recurrent episodes of sneezing. Physical examination and a sinus $x$ ray were unremarkable. He was empirically treated with a 2 week course of oxytetracycline $100 \mathrm{mg}$ twice daily without improvement. Culture of retrograde deep nasal flushings performed under general anaesthesia yielded a profuse growth of Pseudomonas aeruginosa. Tests for feline herpes virus-1, feline calicivirus and cultures for fungal pathogens were negative. PCR diagnostic tests ${ }^{1}$ and tube array genomic fingerprinting $^{2}$ both identified multiple $P$ aeruginosa isolates as the Liverpool Epidemic Strain (LES) (fig 1), a strain that had chronically infected the cat's owner, a patient with cystic fibrosis (CF), for over 10 years. Each of 18 LES isolates tested was sensitive to marbofloxacin, ceftazidime, piperacillin-tazobactam, colistin, meropenem and tobramycin. Although ceftazidime resistance has been reported as a feature of the LES, LES isolates have been shown to be intermittently sensitive to ceftazidime. ${ }^{3}$ Following treatment with marbofloxacin $10 \mathrm{mg}$ once daily for 3 weeks, the symptoms recurred. Repeat nasal washings confirmed the presence of the LES. A further 6 week course of marbofloxacin transiently improved the symptoms, but the cat continues to experience recurrent paroxysmal sneezing episodes and intermittent mucopurulent nasal discharge. At 4 months, a repeat throat swab confirmed the continuing presence of the LES.

The owner was a 54-year-old male with CF (DF508/DF508) who had been chronically infected with LES (identified by genomic fingerprinting) which was intermittently sensitive to ceftazidime. Since 2000 his lung function has been stable (forced expiratory volume in $1 \mathrm{~s} 60 \%$ predicted) and he only requires an average of one course of oral antibiotics per year for pulmonary exacerbations.
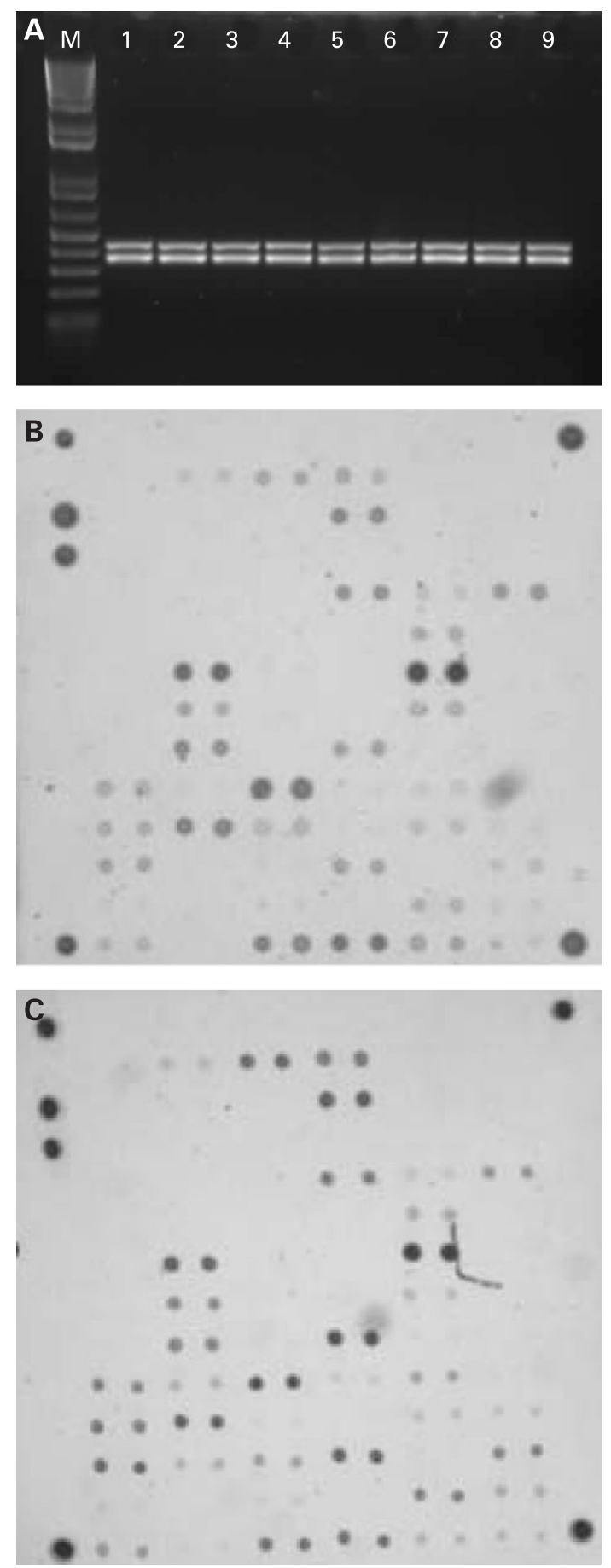

Figure 1 Identification of bacterial isolates as the Liverpool Epidemic Strain (LES). PCR amplification (A) of two LES strain markers from an LES control (lane 1) and eight isolates from the cat (lanes 2-9). M, 1 kb-plus size marker (Invitrogen). Clondiag tube array genetic fingerprinting analysis of a cat isolate (B) and an LES control strain (C) showed identical profiles. 
In between these, he is well with little daily sputum production and rarely carries out chest physiotherapy. The owner lives in a small flat: the cat is an indoor pet and had been living in close contact with his owner since he was 13 weeks old, having free rein of the dwelling space.

\section{DISCUSSION}

Chronic infection with $P$ aeruginosa is common in $\mathrm{CF}$, occurring in up to $80 \%$ of adults. While most patients are infected with unique strains, some strains can be passed between CF patients. Of these, the LES is highly transmissible and is common throughout UK CF clinics. ${ }^{4}$ We have shown that it can "superinfect" patients with CF who already possess previously well established strains of $P$ aeruginosa $a^{5}$ it can infect the relatives of patients with $\mathrm{CF}$, with subsequent chronic morbidity, ${ }^{6}$ and is associated with a poorer prognosis. ${ }^{7}$ Although cross infection of bacteria (methicillin resistant Staphylococcus aureus), fungi and viruses (H5N1) can occur between humans and pets, ${ }^{8}$ this is the first report of interspecies cross infection of a highly transmissible $P$ aeruginosa strain.

Respiratory tract infections are a common cause of morbidity and mortality in cats. Although a number of bacteria have been isolated from the airways in healthy cats, ${ }^{9} P$ aeruginosa is an uncommon cause of respiratory tract infection. ${ }^{10}$ In experimental cat models, repeated $P$ aeruginosa infection causes chronic bronchiolitis and pneumonia, with similar histological features to chronic $P$ aeruginosa infection in human CF. ${ }^{11}$ In this case, we excluded the two most common causes of feline chronic rhinitis (herpesvirus-1 and calicivirus), and it is likely that the chronic respiratory tract infection in the cat by $P$ aeruginosa was due to transmission from the owner, via infected sputum or aerosol spread. Although this individual was not a copious sputum producer nor did he have productive rhinitis, the cat cohabited in a small living space as an indoor pet with ample opportunity for cross infection to occur.
Cross infection with virulent organisms is an increasing problem in the CF community, necessitating the adoption of stringent patient segregation. This report suggests that the LES may have a greater potential for transmission than previously contemplated, further complicating infection control measures for this patient group.

Competing interests: None.

Patient consent: Obtained.

\section{REFERENCES}

1. Smart CHM, Walshaw MJ, Hart CA, et al. Use of suppression subtractive hybridisation to examine the accessory genome of the Liverpool cystic fibrosis epidemic strain of Pseudomonas aeruginosa. J Med Microbiol 2006;55:677-88.

2. Wiehlmann L, Wagner G, Cramer N, et al. Population structure of Pseudomonas aeruginosa. Proc Natl Acad Sci USA 2007;804:8101-6.

3. Fothergill JL, Panagea $S$, Hart CA, et al. Widespread pyocyanin over-production among isolates of a cystic fibrosis epidemic strain. BMC Microbiol 2007; 7:45

4. Scott FW, Pitt TL. Identification and characterisation of transmissible Pseudomonas aeruginosa strains in cystic fibrosis patients in England and Wales. J Med Microbiol 2004;53:609-15.

5. McCallum SJ, Corkill J, Gallagher M, et al. Superinfection with a transmissible strain of Pseudomonas aeruginosa in adults with cystic fibrosis chronically colonized by $P$. aeruginosa. Lancet 2001;358:558-60.

6. McCallum SJ, Gallagher MJ, Corkill JE, et al. Spread of an epidemic Pseudomonas aeruginosa strain from a patient with cystic fibrosis to non-CF relatives. Thorax 2002:57:559-60.

7. Al-Aloul M, Crawley J, Winstanley C, et al. Increased morbidity associated with chronic infection by an epidemic Pseudomonas aeruginosa strain in CF patients. Thorax 2004;59:334-6.

8. Weese JS, Kruth SA. Pets in voluntary household quarantine. Emerg Infect Dise 2006;12:1029-30.

9. Johnson LR, Foley JE, De Cock HEV, et al. Assessment of infectious organisms associated with chronic rhinosinusitis in cats. JAVMA 2005;227:579-85.

10. Schulz BS, Wolf G, Hartman. Bacteriological and antibiotic sensitivity test results in 271 cats with respiratory tract infections. Vet Rec 2006;158:269-70.

11. Thomassen MJ, Klinger JD, Winnie GB, et al. Pulmonary cellular response to chronic irritation and chronic Pseudomonas aeruginosa pneumonia in cats. Infect Immun 1984; $45: 741-7$ 